\title{
NOUVELle
}

\section{Poliovirus et entérovirus C, même espèce, même «tribu » virale}

Mala Rakoto-Andrianarivelo, Sophie Jegouic, Maël Bessaud, Francis Delpeyroux

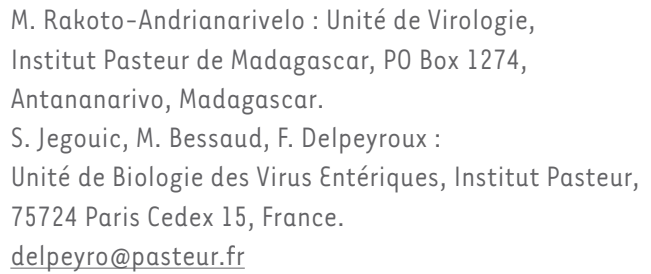

\section{La famille des entérovirus}

Le poliovirus, l'un des virus à ARN les mieux caractérisés est un modèle pour l'étude des virus de la famille des Picornaviridae et du genre Entérovirus auxquels il appartient. Les entérovirus humains, plus de 68 sérotypes connus, sont classés selon leurs degrés de parenté génétique et certaines propriétés biologiques, en cinq espèces distinctes, les entérovirus humains des espèces $A$ à $D$ (HEV-A à -D) et les poliovirus. La particularité des HEV-C (plusieurs sérotypes de coxsackievirus $A$ ) est de présenter des degrés élevés de parenté génétique avec les poliovirus et d'appartenir au même groupe phylogénétique [1].

Les entérovirus sont des virus non enveloppés dont le génome est une molécule d'ARN de polarité positive protégée par une capside de symétrie icosaèdrique. Virus à transmission essentiellement féco-orale, ils se multiplient abondamment dans le tractus digestif et plus particulièrement dans l'intestin grêle. Ils peuvent dans un nombre de cas limité ( $<1 \%$ des cas) se multiplier dans d'autres organes et provoquer alors des pathologies diverses comme herpangines, cardiomyopathies, méningites aseptiques ou encéphalites. En ce qui concerne le poliovirus, il peut atteindre le système nerveux central dans lequel il détruit les neurones moteurs, engendrant la poliomyélite paralytique aiguë.

\section{Vaccin oral contre la poliomyélite} et émergence de souches pathogènes Deux vaccins trivalents permettent de contrôler cette maladie: le vaccin polio injectable (VPI) constitué de virus tués et le vaccin polio oral (VPO) composé de souches virales atténuées qui ont gardé la capacité de se multiplier dans le tractus intestinal des vaccinés mais qui sont dépourvues de pouvoir neuropathogène. En 1988, l'OMS a développé un programme d'éradication de la poliomyélite basé sur des campagnes de vaccination avec le VPO, afin de stopper la circulation des souches sauvages de poliovirus. Ces campagnes ont effectivement permis d'éliminer les poliovirus sauvages dans la plupart des régions du monde puisque, aujourd'hui, ces virus restent endémiques dans quatre pays seulement (Inde, Afghanistan, Pakistan et Nigéria). Cependant, la variabilité et l'évolution rapide des entérovirus pourraient rendre l'éradication du poliovirus plus problématique qu'il n'était prévu initialement. L'emploi du VPO présente, en effet, un inconvénient connu depuis longtemps : les souches vaccinales atténuées révertent très fréquemment vers un phénotype neuropathogène lors de leur multiplication dans le tractus intestinal [2]. Ces souches peuvent provoquer, dans de rares cas (1/700000 primo-doses), une poliomyélite paralytique associée à la vaccination. Un fait nouveau lié à l'emploi du VPO menace aujourd'hui l'objectif d'éradication de la poliomyélite. En effet, des données récentes révèlent que des souches pathogènes dérivées du VPO (appelées VDPV, vaccine-derived polioviruses) sont capables de circuler et d'entretenir un réservoir de souches pathogènes dans des populations où la couverture vaccinale est insuffisante, et de provoquer des épidémies de poliomyélite. Dix épidémies de ce genre ont été détectées ces dernières années essentiellement dans des pays en voie de développement où la poliomyélite due au virus sauvage avait disparu depuis plusieurs années $[3,4]$. Ainsi deux épidémies de poliomyélite provoquées par des VDPV ont été rapportées en 2001-2002 et 2005, à Madagascar alors qu'aucun cas de maladie due au poliovirus sauvage n'avait été détecté dans l'île depuis $1998[5,6]$.

\section{Recombinaison génétique entre souche vaccinale}

de poliovirus et entérovirus HEV-C

Il est remarquable de constater que les VDPV impliqués dans la plupart des épidémies, comme ceux isolés à Madagascar, sont des virus recombinants entre des souches du VPO et des souches non identifiées de poliovirus sauvage ou, plus probablement, d'HEV-C. Les VDPV isolés au cours de l'épidémie de 2001-2002 à Madagascar présentent une structure génomique similaire : des séquences de souches vaccinales dans la moitié 5 '-terminale de leur génome (région 5 ' non-codante et région des protéines structurales) et des séquences de souches d'entérovirus non identifiés dans la moitié 3 '-terminale (protéines non structurales et région 3' non-codante) (Figure 1) [7]. Les génomes des VDPV isolés dans l'île au cours de l'épidémie de 2005 ont une structure plus complexe constituée de séquences provenant de différent sérotypes de poliovirus vaccinal et d'HEV-C divers [5].

Nous avons recherché et caractérisé les entérovirus présents dans les selles de 316 enfants sains, collectées quelques semaines après l'épidémie de 2002 dans les quelques villages où les cas de poliomyélite à VDPV avaient été rapportés [7]. Les résultats indiquent qu'une grande proportion des enfants $(21 \%)$ excrétaient des entérovi- 
rus non poliovirus et en particulier des HEV-C (16\%). Ainsi cinq des six sérotypes d'HEV-C connus pour se multiplier dans des cellules en culture ont été isolés. Le séquençage partiel des génomes des entérovirus isolés a permis d'identifier deux types d'isolats d'HEV-C (coxsackievirus $A 17$ et $A 13$ ) portant des séquences proches des séquences inconnues présentes dans la lignée des VDPV isolés dans la région (Figure 1). De plus les séquences nucléotidiques de ces coxsackievirus se révélaient relativement proches de celles des derniers poliovirus sauvages isolés dans l'île. Ces résultats indiquent que les deux types de coxsackievirus identifiés sont des partenaires privilégiés du poliovirus pour la recombinaison génétique. Par ailleurs, une vingtaine de génotypes d'HEV-C recombinants différents ont été identifiés indiquant que la recombinaison entre sérotypes et/ou souches distinctes d'HEV-C est également un mode d'évolution très fréquent. Ce mécanisme contribue à entretenir au sein de cette petite population d'enfants vivant sur un territoire restreint une biodiversité génétique jamais décrite jusqu'à maintenant dans le monde viral.

\section{Conclusion}

Bien que la recombinaison génétique entre souches de poliovirus vaccinal/vaccinal ou vaccinal/sauvage, et entre échovirus (autres entérovirus) soit un phénomène connu depuis plusieurs années, la signification et les consé- quences biologiques de ces échanges génétiques restent largement incomprises $[2,8,9]$. Ces épidémies à VDPV suggèrent fortement que les remaniements génétiques pourraient jouer un rôle primordial dans l'évolution des entérovirus d'un même groupe et dans l'acquisition d'un phénotype viral nouveau. La densité et la biodiversité des HEV-C aperçues sur le lieu de l'étude laissent penser que cet écosystème viral pourrait fonctionner comme un «chaudron» particulièrement favorable à l'émergence de nouvelles souches d'entérovirus pathogènes, comme les VDPV [7, 10]. Les échanges génétiques fréquents entre poliovirus et $\mathrm{HEV}-\mathrm{C}$, que confirment nos études sur les VDPV, justifient les propositions des taxonomistes qui visent à classer ces deux types viraux au sein de la même espèce virale des HEV-C [1]. Plus surprenant est de constater que ces virus peuvent co-exister, co-circuler, évoluer et se diversifier dans certaines régions du monde au sein d'une même «tribu» virale. $\diamond$

Polioviruses and species C enteroviruses, viruses

of the same species and « tribe »

\section{REMERCIEMENTS}

Nous remercions pour leur soutien, la Direction des Affaires Internationales et la Direction des Programmes Transversaux de Recherche (PTR 120 et PTR 276) ainsi que le Ministère des Affaires Etrangères (FSP 2001-168). S.J. est boursière de la Délégation Générale pour l'Armement et du Centre National pour la Recherche Scientifique (CNRS).

\section{RÉFÉRENCES}

1. Brown B, Oberste MS, Maher K, et al. Complete genomic sequencing shows that polioviruses and members of human enterovirus species $C$ are closely related in the noncapsid coding region. J Virol $2003 ; 77: 8973-84$.

2. Agol VI. Molecular mechanisms of poliovirus variation and evolution. Curr Top Microbiol Immunol 2006 ; 299 : 211-59.

3. Kew OM, Sutter RW, De Gourville EM, et al. Vaccine-derived polioviruses and the endgame strategy for global polio eradication. Annu Rev Microbiol 2005 ; 59 : 587-635

4. World Health Organization. Global update on vaccinederived polioviruses, January 2006-August 2007. Weekly Epidemiol Rec 2007 ; 82 : 337-44.

5. Rakoto-Andrianarivelo M, Gumede N, Jegouic S, et al. Re-emergence of recombinant vaccine-derived poliovirus outbreak in Madagascar. J Infect Dis 2008 (sous presse).

6. Rousset $D$, Rakoto-Andrianarivelo M, Razafindratsimandresy $\mathrm{R}$, et al. Recombinant vaccine-derived poliovirus in Madagascar. Emerg Infect Dis 2003; $9: 885-7$.

7. Rakoto-Andrianarivelo M, Guillot $S$, Iber $S$, et al. Cocirculation and evolution of polioviruses and species $C$ enteroviruses in a district of Madagascar. PLoS Pathog 2007; 3 : el91.

8. Cuervo N, Guillot S, Romanenkova N, et al. Genomic features of intertypic recombinant Sabin strains excreted by primary vaccinees. J Virol $2001 ; 75: 5740-51$.

9. Guillot S, Caro V, Cuervo N, et al. Natural genetic exchanges between vaccine and wild poliovirus strains in humans. J Virol $2000 ; 74: 8434-43$.

10. Rakoto-Andrianarivelo M, Rousset D, Razafindratsimandresy $R$, et al. High frequency of human enterovirus species C circulation in Madagascar. J Clin Microbiol 2005 ; 43 : 242-9.

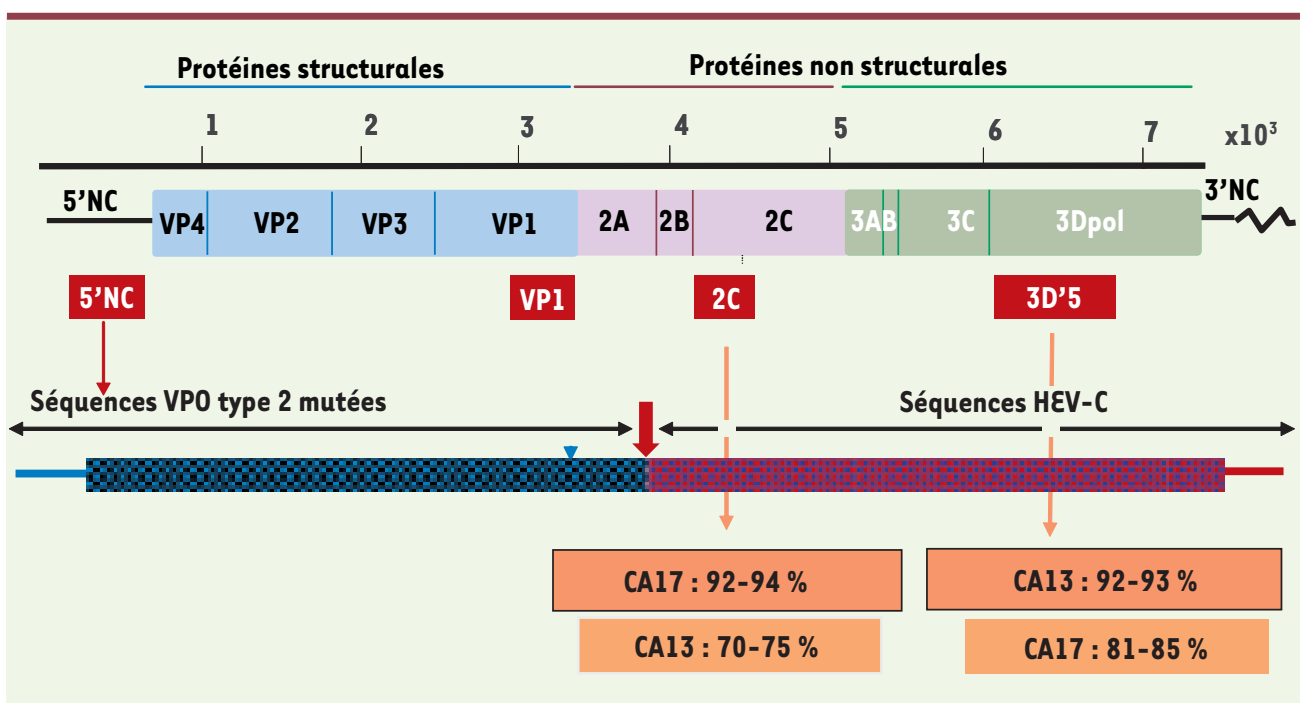

Figure 1. Structure génomique d'un VDPV recombinant isolé à Madagascar. L'organisation du génome des entérovirus est indiquée avec les régions 5' - et 3 '-non codantes ( $5^{\prime} \mathrm{NC}$ et $\left.3^{\prime} \mathrm{NC}\right)$. La phase ouverte de lecture codant les protéines de la capside du virion (VPI à VP4) et les protéines impliquées dans la multiplication virale (2A à 3Dpol) ainsi que la taille du génome viral (x $10^{3}$ nucléotides) sont représentés. La séquence nucléotidique du génome du VDPV ainsi que les séquences partielles de quatre régions génomiques ( $5^{\prime} \mathrm{NC}, \mathrm{VPl}, 2 \mathrm{C}$ et 3D'5) de tous les entérovirus non poliovirus co-circulant avec le VDPV ont été déterminées. Le génome du VDPV apparaît

formé de séquences dérivées de la souche vaccinale de type 2 du VPO et de séquences d’HEV-C, le site de recombinaison étant localisé dans la protéine virale $2 A$ (flèche rouge). Les séquences d'HEV-C apparaissent proches des séquences de souches de coxsackievirus A17 (CA17) dans la région 2C et de souches de coxsackievirus Al3 (CAl3) dans la région 3Dpol. Les pourcentages d'identité nucléotidique entre le VDPV et les souches de coxsackievirus sont indiqués. 